\title{
Stratégies et dynamiques territoriales à Paris dans le champ de la santé de la mère et du jeune enfant
}

\section{Béatrice Georgelin}

\section{OpenEdition}

Journals

Édition électronique

URL : http://journals.openedition.org/rfst/603

DOI : $10.4000 /$ rfst.603

ISSN : 2492-3672

\section{Éditeur}

Espaces et SOciétés (UMR 6590)

\section{Référence électronique}

Béatrice Georgelin, «Stratégies et dynamiques territoriales à Paris dans le champ de la santé de la mère et du jeune enfant », Revue francophone sur la santé et les territoires [En ligne], Ateliers et séminaires, mis en ligne le 25 novembre 2016, consulté le 06 avril 2021. URL : http:// journals.openedition.org/rfst/603; DOI : https://doi.org/10.4000/rfst.603

Ce document a été généré automatiquement le 6 avril 2021.

\section{(i) 8 (2)}

La Revue francophone sur la santé et les territoires est mise à disposition selon les termes de la Licence Creative Commons Attribution - Pas d'Utilisation Commerciale - Partage dans les Mêmes Conditions 4.0 International. 


\title{
Stratégies et dynamiques territoriales à Paris dans le champ de la santé de la mère et du jeune enfant
}

\author{
Béatrice Georgelin
}

1 L'amélioration des parcours de santé des femmes durant leur grossesse et des jeunes enfants est un objectif général de santé publique mobilisant de nombreux acteurs, à la fois institutionnels (État, Agences Régionales de Santé, Conseils Départementaux), professionnels (maternités, services de Protection Maternelle et Infantile, professionnels de santé libéraux) ainsi que d'autres acteurs de terrain, notamment associatifs.

2 Il se décline notamment en un objectif de réduction des inégalités socio-territoriales dans l'accès et la qualité du suivi proposé aux femmes et aux familles, objectif politique partagé par l'ensemble des acteurs publics de l'offre de santé dans ce champ ${ }^{1}$. Pour l'atteindre, l'enjeu ne consiste pas seulement à optimiser la prise en charge actuelle, mais de réfléchir à une évolution des modes d'actions et à l'articulation des interventions de ces multiples acteurs, au plus près des besoins locaux. L'étude des reconfigurations territoriales de ce système d'acteurs permet de mettre en lumière les tensions et les limites des dynamiques en cours en regard de l'objectif de réduction des inégalités.

3 À Paris, cet objectif se traduit par un effort porté sur l'amélioration de la prise en charge des femmes et des familles les plus vulnérables, qui n'ont accès qu'à l'offre publique ou associative, effort conduit par la collectivité parisienne, au titre des compétences de Protection Maternelle et Infantile (PMI) du Département de Paris, et de nombreux partenaires du soin, de la prévention et de l'accompagnement.

Cette communication vise ainsi à :

- Présenter les acteurs de ce champ à Paris, à travers leurs différentes missions, ressources, stratégies et territorialités (Vanier, 2009). 
- Montrer la pluralité et la complémentarité des stratégies territoriales mises en œuvre pour répondre aux besoins des plus vulnérables.

- Interroger les effets et les fragilités de ce système d'acteurs, dans un contexte actuel de pressions budgétaires croissantes. qu'objectif qui est à interroger, dans la mesure où les dynamiques observées à Paris témoignent d'un enjeu plus fondamental : l'adaptation de l'offre de santé afin qu'elle soit réellement accessible à tous.

$6 \quad$ Matériaux et méthode . Les résultats présentés ici sont issus de recherches menées dans le cadre d'une thèse portant sur les processus de territorialisation à l'œuvre dans l'action publique locale en santé à Paris, qui s'appuie sur une observation participante dans différentes démarches de "démocratie sanitaire " portées par la Mairie de Paris, en 2015 et 2016 ainsi que sur des entretiens réalisés auprès d'acteurs en santé, principalement publics. Ont été mobilisés pour cette présentation :

- Des données de terrain produites lors de la participation aux débats publics et aux ateliers des États Généraux de la PMI de Paris, démarche de concertation organisée en 2015. En particulier, celles recueillies lors des réunions de l'atelier «La PMI active dans les territoires » auxquelles participaient des professionnels de la PMI de Paris et leurs partenaires : Ateliers Santé Ville parisiens (ASV), professionnels du social et médico-social, chercheurs, ...

- Des entretiens réalisés en 2015 et 2016 auprès de professionnels et d'administrateurs de la PMI de Paris; de professionnels de l'Agence Régionale de Santé (ARS) d'Ile-de-France en charge de la périnatalité ; du réseau de santé périnatale parisien ; d'acteurs associatifs...

\section{Contexte sociodémographique et sanitaire}

7 Paris présente une natalité importante et les principaux indicateurs en santé périnatale y sont plutôt bons, proches des taux moyens régionaux et nationaux (Vincelet et al., 2016) ${ }^{2}$. Toutefois, des disparités territoriales infra-parisiennes, parfois importantes, peuvent être observées. Pour le suivi des grossesses, on observe par exemple des variations importantes entre arrondissements sur certains indicateurs clés, comme la proportion de femmes n'ayant pas réalisé l'ensemble des échographies recommandées durant leur grossesse ou le pourcentage de femmes déclarant tardivement leur grossesse (DASES, 2015).

travaux issus de l'enquête nationale périnatale ont montré la persistance de disparités sociales et territoriales en France en ce qui concerne les parcours de santé des mères et des jeunes enfants, et in fine les indicateurs sanitaires liés (Blondel, Kermarrec, 2011). De nombreuses vulnérabilités sont à prendre en compte pour expliquer ces disparités : vulnérabilités affectives et personnelles, vulnérabilités liées aux multiples formes de précarité, aux conditions de vie, de travail et de logement, aux ressources familiales et amicales, aux parcours de vie de ces femmes, notamment aux parcours de migrations... (Gasquet, 2013).

9 Les disparités observées à Paris participent ainsi plus largement d'inégalités socioterritoriales de santé. La féminisation de la précarité, en particulier de la grande exclusion, l'aggravation de la précarité et la forte proportion de familles étrangères, pour certaines migrantes, sont, parmi cet ensemble de vulnérabilités et d'inégalités, les

Revue francophone sur la santé et les territoires , Ateliers et séminaires 
problématiques particulièrement saillantes aux yeux des professionnels de terrain à Paris.

\section{Missions et problématiques des différents acteurs de l'offre de santé}

Différents acteurs publics assurent la prise en charge médicale, l'offre de prévention et d'accompagnement des femmes tout au long des parcours de grossesse, puis des enfants après leur naissance.

En comparaison d'autres départements d'Ile-de-France, où peuvent être observés des problèmes de couverture territoriale, cette offre de santé est importante et diversifiée à Paris. Différents enjeux selon ces acteurs se posent alors.

2 Les maternités parisiennes sont de plus en plus poussées à développer des réseaux professionnels pour améliorer la prise en charge des grossesses à risques et assurer le suivi prénatal des grossesses en ville. Bien que persiste encore aujourd'hui une certaine méconnaissance de la part des maternités de ce qui se passe autour d'elles, sous l'effet de pressions budgétaires et de différents leviers comme la contractualisation avec l'ARS, elles sont incitées à mieux connaître les ressources environnantes et développer des partenariats avec ces acteurs hors du soin.

La PMI de Paris compte 62 centres, ouverts à toutes les familles. Le principe d'universalisme proportionné et l'approche globale et préventive au fondement de ce service public conduisent la PMI à chercher à s'adapter aux besoins des familles, notamment des plus vulnérables. Des stratégies partenariales et d'« aller-vers » variées sont développées : déplacements à domicile ou dans certains foyers d'hébergement, groupes et ateliers dédiés à certaines populations, offre complémentaire à celle des maternités pour le suivi de grossesse des femmes très précaires... La mise en place de territoires de PMI, regroupant un ou plusieurs arrondissements chacun, doit permettre de constituer ces réseaux partenariaux au plus près du « terrain ».

14 Toutefois, faute de moyens humains suffisants, la PMI de Paris est amenée à suivre en priorité les familles les plus défavorisées (sans couverture sociale, vivant en centre d'hébergement ou en hôtel social,...) et à rediriger les familles plus aisées vers l'offre libérale. Certains centres de PMI reçoivent ainsi en majorité des familles cumulant les vulnérabilités, ce qui rend l'accompagnement plus complexe. Il en résulte une tension entre la recherche permanente d'adaptation de la PMI et l' « embolie » des services.

D'autres acteurs institutionnels ou de terrain interviennent en complément de ces deux acteurs principaux de l'offre de soins et de prévention.

L'ARS Ile-de-France finance directement différents réseaux de coordination : le réseau de santé périnatale parisien, d'autres réseaux régionaux thématiques couvrant Paris (femmes migrantes, nouveaux nés prématurés). Et dans le cadre du fond d'intervention régional, elle finance des projets "périnatalité et petite enfance", portés à Paris exclusivement par des associations soit situées dans un des quartiers prioritaires de la politique de la ville, soit travaillant auprès de publics en très grande précarité.

Des dispositifs comme les Ateliers Santé Ville (ASV) parisiens, implantés eux aussi dans les quartiers prioritaires de la politique de la ville, joue un rôle en œuvrant à la coordination d'acteurs sur le thème de la santé des femmes, notamment par la 
production d'annuaires, la mise en place de groupes de travail ou l'appui de projets locaux.

Enfin, des structures et des associations du champ social et médico-social, comme les centres sociaux, peuvent porter, seules ou conjointement, des projets pour améliorer l'accompagnement et la prise en charge des femmes et des familles. Le projet d'accompagnement de la maternité et à l'allaitement porté par Emmaüs Solidarité un projet, en partenariat avec la maternité de Port-Royal dans l'un de ses Espaces Insertion Famille en est un exemple (projet financé par l'ARS). Ces démarches ascendantes sont très souvent ancrées dans un territoire et partent de difficultés identifiées à cet endroit.

Carte 1 : Implantation géographique des différents acteurs publics dans le champ de la santé des mères et des jeunes enfants à Paris mentionnés dans le résumé.

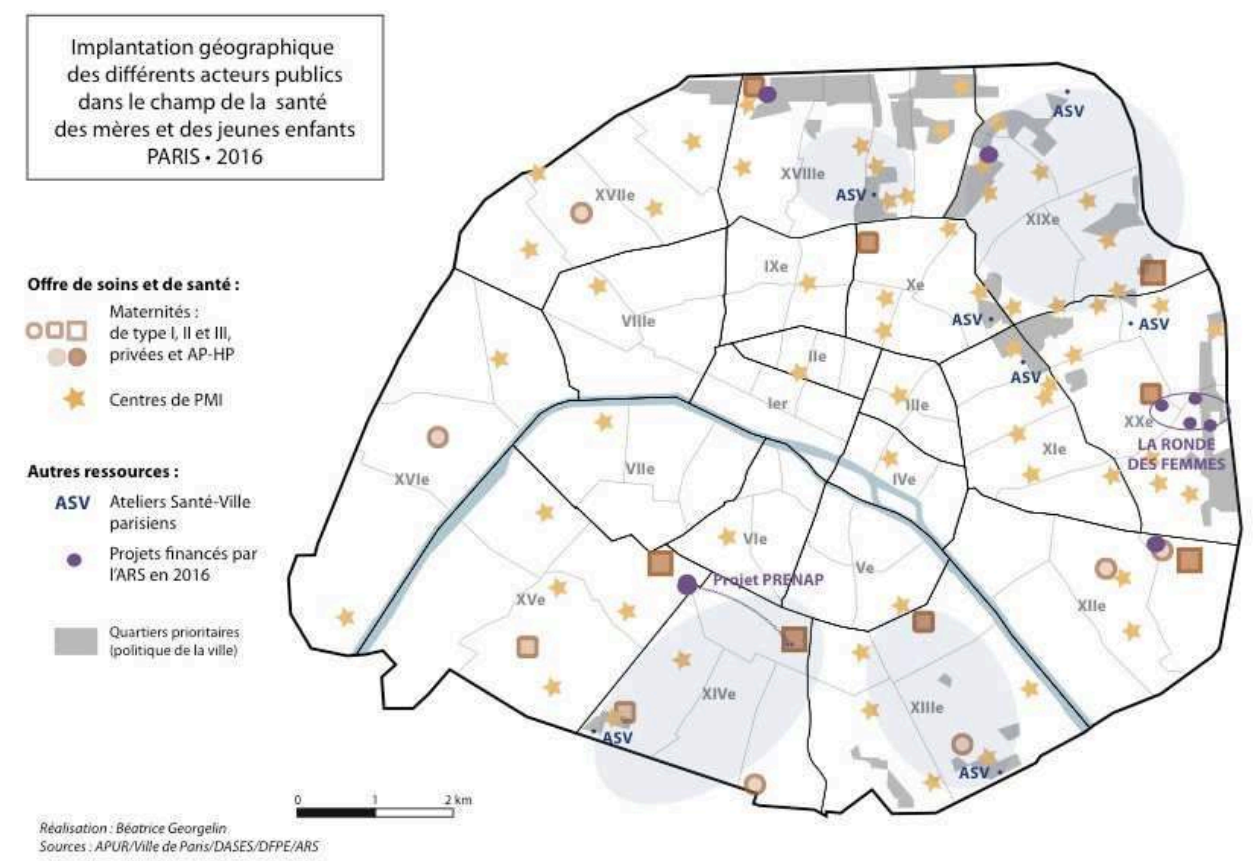

\section{Reconfigurations territoriales de l'action en santé : espaces, territoires et lieux}

Plus que la réduction globale des inégalités, c'est l'amélioration de la prise en charge des personnes les plus précaires et les plus vulnérables qui est le moteur commun de dynamiques plurielles, opérant à différentes échelles.

Logiques de spatialisation des ressources . À l'échelle parisienne, tant l'ARS d'Ile-deFrance que la Ville de Paris mettent en œuvre des politiques qui s'appuient sur des stratégies de spatialisation (Dubresson et Jaglin, 2005), veillant à ce que leurs ressources soient prioritairement allouées sur certains territoires parisiens, visant ainsi les populations les plus défavorisées. Les critères de priorisation territoriale pour l'attribution des crédits de l'ARS conduisent au ciblage exclusif de ces financements sur les quartiers prioritaires politique de la ville à Paris et/ou sur les personnes en situation de très grande exclusion. L'implantation géographique des centres de PMI et 
leur répartition sur Paris est perçue comme pertinente pour les directeurs de la PMI, les centres étant plus nombreux dans le nord-est parisien où la natalité est la plus forte et la part des familles vulnérables est la plus importante.

21 Émergence et implantation de territoires d'action . La forte densité d'acteurs sur Paris s'accompagne d'un manque de connaissance réciproque de ces acteurs, de leurs apports et de leurs fonctionnements. Les professionnels de terrain expriment fréquemment le besoin de davantage de mise en réseau et sont par ailleurs de plus en plus fortement enjoints à participer à des coordinations pluri-professionnelles. Ce besoin de coordination s'exprime à différentes échelles : tant au niveau parisien, qu'au niveau des arrondissements, qu'à un niveau plus local encore. Certains réseaux et projets locaux existants à Paris peuvent ainsi s'apparenter à de véritables « constructions locale de santé » (Fleuret, 2012), s'agissant de démarches portées par quelques acteurs implantés dans un territoire. Le projet « La Ronde des Femmes » dans le 20e, porté conjointement par plusieurs centres sociaux de l'arrondissement et en partenariat avec la maternité de l'hôpital Tenon, en est l'illustration: alors que certaines femmes venaient accoucher dans cette maternité sans trousseau, un projet associant plusieurs centres sociaux et leurs ressources propres s'est mis en place pour accompagner ces femmes dans la parentalité et l'accueil de l'enfant.

Réflexion autour des lieux où favoriser l'accès à l'offre de santé, notamment de prévention . Les réflexions autour de l'« aller-vers » et du décloisonnement de la santé appuient différentes initiatives : équipes mobiles, permanences de professionnels de santé dans les structures sociales, les accueils de jour, les centres d'hébergement, groupes de parole et accompagnement dans les structures associatives... Une pluralité de lieux autres que les maternités et les centres de PMI peuvent être ainsi des lieux favorables à la santé et au bien-être des femmes enceintes.

Les modes d'actions possibles en ce sens, et les moyens disponibles pour, furent au cœur des débats des États Généraux de la PMI, un médecin de PMI ayant ainsi souligné la faiblesse de ces moyens: "La PMI hors les murs? Mais il n'y a personne dans les murs !». Pour d'autres acteurs institutionnels se pose également la question du rapport coût/bénéfice, mesuré en terme de moyens humains alloués pour le nombre de personnes touchées par action, des actions menées dans des centres ou des foyers. Bien qu'elles permettent de toucher des personnes qui ne le sont pas par des campagnes de prévention universelles, elles sont beaucoup plus couteuses et donc fragiles, dans un contexte de réduction des effectifs et de diminution des enveloppes budgétaires.

Une certaine complémentarité entre ces stratégies apparaît, ainsi qu'une forte interdépendance des acteurs qui les portent, entre financeurs et opérateurs par exemple.

\section{Portée et limites : quelle offre de santé pour tous ?}

La diversification des lieux et des approches, l'élaboration de projets locaux s'appuyant sur des dynamiques partenariales inscrites dans leur territoire, sont autant de leviers pour améliorer les parcours de santé de certaines femmes et familles, éloignées de la prise en charge proposée par un service public universel et généraliste.

Néanmoins, on peut souligner la concentration d'une part importante des ressources publiques sur certains quartiers et en direction de certains publics, les plus vulnérables. 
Cela soulève la question de la spécialisation de l'action publique et de l'impact que cela peut avoir sur les organisations professionnelles, notamment la PMI. Par ailleurs, on peut observer des effets pervers classiques quant aux zonages retenus pour conduire cette priorisation, qui prennent parfois difficilement en compte les territoires vécus par les habitants. Cela soulève également la question des moyens publics alloués pour la santé de toutes et des dynamiques à l'œuvre dans les autres territoires, non prioritaires. Enfin, face à des inégalités socio-territoriales de santé persistantes, ces stratégies sont-elles appelées à devenir des organisations définitives?

Par ailleurs, l'injonction à davantage de coordination ne résout pas toutes les difficultés posées par l'articulation de différentes approches en santé, d'univers distincts : santé publique, santé communautaire, travail social,...

Les questions du financement et de la pérennité de ce système d'acteurs sont centrales, alors que l'ensemble des financements est à la baisse. Les acteurs non-institutionnels sont des relais indispensables de la politique de santé, mais on ne peut que constater la précarité de leur mode de financement, tant en volume disponible qu'en ce qui concerne les modalités, la temporalité et la prévisibilité de l'accès à ces financements.

En conclusion, deux enjeux principaux traversent cette présentation: les limites que rencontrent services publics et autres dispositifs institutionnels universalistes pour proposer une offre de santé adaptée à tous dans une métropole cosmopolite marquée d'importantes inégalités, et la fragilité du financement des acteurs associatifs, qui assurent pourtant aujourd'hui une part indispensable de l'offre de santé.

\section{BIBLIOGRAPHIE}

BLONDEL B., KERMARREC M., 2011, « La situation périnatale en France en 2010, Premiers résultats de l'enquête nationale périnatale », Drees, Études et résultats, ${ }^{\circ}{ }^{\circ}$ 775, octobre 2011.

COHUET S., DANZON A., HAUSHERR E., ROGER S., 2015, « La santé des mères et des nouveaux nés, Données issues des premiers certificats des enfants nés en 2013 et domiciliés à Paris », DASES, Ville de Paris, en partenariat avec l'Atelier Parisien d'Urbanisme.

DUBRESSON A., JAGLIN S., 2005, « Gouvernance, régulation et territorialisation des espaces urbanisés : Approches et méthode », dans ANTHEAUME B., GIRAUT F. (dir.), Le territoire est mort, vive les territoires! : une (re)fabrication au nom du développement, Paris, France, IRD éd.

GASQUET C., 2013, « Itinéraires de santé liés au suivi et à l'issue de grossesse », étude réalisée avec la participation de $\mathrm{M}$. Collombier et d'A-C Hoyez, présentée à l'occasion des rencontres du réseau SOLIPAM en novembre 2013.

FLEURET S., 2012, Construction locale de santé. Attention primaire et santé communautaire, comparaisons internationales et expérimentation en France., Habilitation à diriger des recherches, Université d'Angers.

VANIER M., 2009, Territoires, territorialité, territorialisation: controverses et perspectives, Rennes, France, Presses Universitaires de Rennes (Entretiens de la Cité des Territoires), 228 p. 
VINCELET C., LEBRETON E., POULAIN C., CRENN-HÉBERT C., MENGUY C., DANIEL AG., 2016, « La santé périnatale en Ile-de-France : Tableau de bord d'indicateurs départementaux et régionaux en périnatalité et orthogénie ", mise à jour Mai 2016. Observatoire régional de santé Ile-deFrance, ARS Ile-de-France. Consultable en ligne à l'adresse suivante $:$ http://www.perinat-arsidf.org

\section{NOTES}

1. Ce champ, désigné comme celui de la santé périnatale, ou de la santé maternelle et infantile, ou encore de la santé de la mère et du jeune enfant, recouvre différentes questions de santé liées à la grossesse (planification familiale, suivi des grossesses normales, prise en charge médicale des grossesses à risques), à l'accouchement, au suivi du développement et de la santé du jeune enfant de 0 à 3 ans.

2. Environ 29000 bébés parisiens naissent chaque année depuis 2011, le taux de natalité étant plus élevé dans les arrondissements du nord et nord-est parisien. La proportion élevée de mères étrangères à Paris est également à noter : ces dernières années, en moyenne, $30 \%$ des bébés parisiens sont nés de mère étrangère, soit deux fois plus que la moyenne nationale.

\section{INDEX}

Mots-clés : mère, enfant, inégalités sociales et territoriales de santé

Index géographique : Paris

\section{AUTEUR}

BÉATRICE GEORGELIN

ESO - CNRS UMR 6590, Université d'Angers 